Mots. Les langages du politique

\title{
Bibliographie thématique (2001-2005)
}

Discours de violence au nom de la foi

\section{(2) OpenEdition}

\section{Journals}

Édition électronique

URL : https://journals.openedition.org/mots/1546

DOI : $10.4000 /$ mots. 1546

ISSN : 1960-6001

Éditeur

ENS Éditions

\section{Édition imprimée}

Date de publication : 1 novembre 2005

Pagination : 83-89

ISBN : 2-84788-084-4

ISSN : 0243-6450

\section{Référence électronique}

«Bibliographie thématique (2001-2005) », Mots. Les langages du politique [En ligne], 79 | 2005, mis en

ligne le 28 mai 2008, consulté le 24 avril 2022. URL : http://journals.openedition.org/mots/1546 ; DOI : https://doi.org/10.4000/mots.1546

\section{(C) ENS Éditions}




\section{Bibliographie thématique (2001-2005). Discours de violence au nom de la foi}

ABBÈS M., 2003, «Guerre et paix en islam : naissance et évolution d'une théorie », Mots. Les Langages du politique, $\mathrm{n}^{\circ} 73$, novembre, Les discours de la guerre, p. 43-58.

ABD EL MOTALEB AL HOUNI M., 2004, L'impasse arabe. Les Arabes face à la nouvelle stratégie américaine, préfacé et traduit par L. Lakhdar, Paris, L'Harmattan, 112 p.

AL-AZMEH A., 2004, L'obscurantisme postmoderne et la question musulmane, traduit de l'anglais parY. Jraissati, Paris, Sindbad Actes Sud, $60 \mathrm{p}$.

AL-QARADHAWIY., 2002, La Sunna du Prophète. Réflexion sur notre approche de la Sunna, Paris, Al-Qalam, $240 \mathrm{p}$.

AMIR-MOEZZI M., JAMBERT C., 2004, Qu'est-ce que le shîiisme ?, Paris, Fayard, 408 p.

AMIRPUR K., 2003, L'Iran des réformes. Avec Shirin Ebadi, prix Nobel de la paix, Paris, Alvik, $192 \mathrm{p}$.

BALENCIE J.-M., DE LA GRANGE A. éd., 2004, Les nouveaux mondes rebelles. Conflits, terrorisme et contestations, Paris, Michalon, $503 \mathrm{p}$.

BARILIER E., 2004, Nous autres civilisations... Amérique, Islam, Europe, Carouge, Genève, Zoé.

BARRADA H., SITBON G., 2004, L'Arabe et le Juif. Dialogue de guerre, Paris, Plon, $271 \mathrm{p}$.

BAER R., 2002, La chute de la CIA. Les mémoires d'un guerrier de l'ombre sur les fronts de l'islamisme, Paris, Lattès, $392 \mathrm{p}$.

BANNELIER K., CHRISTAKIS Th., CORTEN O., DELCOURT B. éd., 2002, Cahiers internationaux, $\mathrm{n}^{\circ} 17$, Le droit international face au terrorisme.

BAUER A., RAUFERX., 2002, La guerre ne fait que commencer, Paris, Lattès, 320 p.

— 2005, L'énigme Al-Qaida, Paris, Lattès, 345 p.

BENBASSA E., 2004, La République face à ses minorités. Les juifs hier, les musulmans aujourdhui, 2004, Paris, Mille et Une Nuits, $154 \mathrm{p}$.

BEN JELLOUN T., 2004 [1998], Le racisme expliqué à ma fille, Paris, Le Seuil, 63 p.

BEN MANSOUR L., 2002, Frères musulmans, frères féroces. Voyage dans l'enfer $d u$ discours islamiste, Paris, Ramsay, $263 \mathrm{p}$.

— 2004, Les mensonges des intégristes, Paris, Le Serpent à plumes, 265 p.

BENNANI-CHRAÏBI M., FILLIEULE O., 2003, Résistance et protestation dans le monde musulman, Paris, Presses de Sciences Po.

BENTOUNĖS K., HOUZIAUX A., ISRAËL G., 2004, Le Coran, Jésus et le judaïsme, Paris, Desclée de Brouwer, $208 \mathrm{p}$.

BENZINE R., 2004, Les nouveaux penseurs de l'islam, Paris, Albin Michel, 304 p. BERMAN P., 2004, Les habits neufs de la terreur, Paris, Hachette Littérature, $274 \mathrm{p}$. BERNARD-MARIE (Frère OFS), 2002, L'Islam et la miséricorde, Paris, Autrement, 92 p.

BLIN A., CHALIAND G. éd., 2004, Histoire du terrorisme. De l'Antiquité à Al Qaida, Paris, Bayard, $668 \mathrm{p}$. 
BLUM W., 2002, L'État voyou, Paris, Parangon, 364 p.

BONAN C., 2002, Le Soufisme. Al-tasawwuf et la spiritualité islamique, Maisonneuve et Larose, $156 \mathrm{p}$.

BONIFACE P., 2005, Vers la $4^{e}$ guerre mondiale ?, Paris, Armand Colin, $173 \mathrm{p}$.

BONNER M., 2004, Le jihad. Origines, interprétations, combats, Paris, Téraèdre.

BOUBAKEUR A., LAMBERT P., SIBONY D., 2005, Le choc des religions. Juifs, chrétiens, musulmans, la coexistence est-elle possible?, Paris, Presses de la Renaissance, $220 \mathrm{p}$.

BOULET-GERCOURT Ph., 2004, La Machine Bush, Paris, Grasset, 261 p.

BOUNAN M., 2003, Logique du terrorisme, Paris, Allia, $61 \mathrm{p}$.

BOUZAR D., 2001, L'islam des banlieues. Les prédicateurs musulmans: nouveaux travailleurs sociaux, Paris, Syros, $180 \mathrm{p}$.

BRIZARD J.-Ch., DASQUIÉ G., 2002, Ben Laden, la vérité interdite, Paris, Denoël, 328 p.

- (en collab. avec MARTINEZ D.), 2005, Zarkaoui. Le nouveau visage d'Al Qaida, Paris, Fayard, $340 \mathrm{p}$.

BURGAT F., 2002 [1995], L'islamisme en face, Paris, La Découverte, 285 p.

BURKE J., 2005, Al-Qaida. La véritable histoire de l'islam radical, Paris, La Découverte, $316 \mathrm{p}$.

Cahiers de l'Orient éd., 2003, Dictionnaire mondial de l'islamisme, Paris, Plon, 518 p.

Cahiers internationaux, voir BANNELIER.

CARRÉ 0., 2004 [1984, Presses de la FNSP et Le Cerf], Mystique et politique: le Coran des islamistes, réédition revue et complétée par Mystique et politique: lecture révolutionnaire du Coran par Sayyid Qutb, frère musulman radical (1906-1966), Paris, Le Cerf, $400 \mathrm{p}$.

- 2004 [1989], Le nationalisme arabe, Paris, Payot, 322 p.

- SEURAT M . [MICHAUD G.], 2001 [1983, Gallimard-Julliard], Les Frères musulmans, 1928-1982, Paris, L'Harmattan, 252 p.

CESARI J., 2004, L'islam à l'épreuve de l'Occident, Paris, La Découverte, 292 p.

CHARNAY J.-P., 2004, Principes de stratégie arabe, Paris, L'Herne, $650 \mathrm{p}$.

CHEBEL M. éd., 2005, L'islam, passion française. Une anthologie, Paris, Bartillat, 385 p.

— 2004, Pour un islam des Lumières. 27 propositions pour réformer l'islam, Paris, Hachette, $224 \mathrm{p}$.

CHOMSKY N., 2004, Dominer le monde ou sauver la planète? L'Amérique en quête d'hégémonie mondiale, Paris, Fayard, $388 \mathrm{p}$.

Cités, voir ZARKA.

CLARKE R., 2003, Contre tous les ennemis : au cœur de la guerre américaine contre le terrorisme, Paris, Albin Michel, $368 \mathrm{p}$.

COFFLARD M., 2004, L'Émir. La peur aura-t-elle le dessus ?, Paris, Fayard, 327 p.

COMMEAU G., 2004, Grâce à l'autre. Le pluralisme religieux, une chance pour la foi, Paris, L'Atelier, $160 \mathrm{p}$.

COSANDEY F., DESCIMON R., 2002, L'absolutisme en France, Paris, Le Seuil, 319 p.

COURMONT B., 2003, Terrorisme et contre-terrorisme, l'incompréhension fatale, Paris, Le cherche midi, $144 \mathrm{p}$.

DASQUIÉ G., 2005, Al-Qa'ida vaincra, Paris, Flammarion, $310 \mathrm{p}$.

DAWOD H. éd., 2004, Tribus et pouvoirs en terre d'islam, Paris, Armand Colin, 303 p.

DEBRAY R., 2002, Ce que le voile nous voile. La République et le sacré, Paris, Gallimard, $50 \mathrm{p}$.

84 - Discours de violence au nom de la foi 
— 2004, Terrorisme, guerres, diplomatie. Chroniques de l'idiotie triomphante (19902003), Paris, Fayard, 206 p.

DELCAMBRE A.-M., 2003, L'Islam des interdits, Paris, Desclée de Brouwer, 146 p.

DELOIRE C., DUBOIS C., 2004, Les islamistes sont déjà là. Enquête sur une guerre sainte, Paris, Albin Michel, $349 \mathrm{p}$.

DERRIDA J., HABERMAS J., 2004, Le «concept» du 11 septembre. Dialogues à New-York (octobre-décembre 2001) avec Giovanna Borradori, Paris, Galilée, 251 p.

DIAGNE S. B., 2001, Islam et société ouverte. La fidélité et le mouvement dans la pensée de Muhammad lqbal, Paris, Maisonneuve et Larose, $160 \mathrm{p}$.

DJAîT H., 2004, La crise de la culture islamique, Paris, Fayard, 336 p.

DJAVANN C., 2004, Que pense Allah de l'Europe ?, Paris, Gallimard, 56 p.

DOUSSE M., 2002, Dieu en guerre. La violence au coeur des trois monothéismes, Paris, Albin Michel, $256 \mathrm{p}$.

DUPIN É., 2004, L'hystérie identitaire, Paris, Le cherche midi, 168 p.

ENDERLIN Ch., 2004 [1997, Stock], Paix et guerres. Les secrets des négociations israéloarabes, 1917-1995, Paris, Fayard, $778 \mathrm{p}$.

ESACK F., 2004, Coran: mode d'emploi, traduit de l'anglais par J.-L. Bour, Paris, Albin Michel, $346 \mathrm{p}$.

ÉTIENNE B., 2002, Les amants de l'apocalypse. Pour comprendre le 11 septembre, La Tour d'Aigues, Éditions de l'Aube, $71 \mathrm{p}$.

— 2003, Islam, les questions qui fâchent, Paris, Bayard, $130 \mathrm{p}$.

FALLACI 0., 2002, La rage et l'orgueil, Paris, Plon, 196 p.

FATH S., 2002, Billy Graham, pape protestant ?, Paris, Albin Michel, 303 p.

— 2004, Militants de la Bible aux États-Unis. Évangéliques et fondamentalistes du Sud, Paris, Autrement, $220 \mathrm{p}$.

— 2004, Dieu bénisse l'Amérique: la religion de la Maison Blanche, Paris, Le Seuil, $240 \mathrm{p}$.

FAVRET-SAADA J. (en collab. avec CONTRERAS J.), 2004, Le christianisme et ses juifs, de 1800 à 2000 , Paris, Le Seuil, $504 \mathrm{p}$.

FERENCZI Th. éd., 2003, Religion et politique : une liaison dangereuse?, Bruxelles, Complexe, $320 \mathrm{p}$.

FERJANI M.-C., 2004, «Islam, paix et violence », Projet, $\mathrm{n}^{\circ}$ 281, juillet, p. 47-52.

FILASI-ANSARY A., 2002 [1997], L'islam est-il hostile à la laïcité ?, Paris, Sindbad Actes Sud.

— 2003, Réformer l'islam ? Une introduction aux débats contemporains, Paris, La Découverte, $283 \mathrm{p}$.

— 2003, "Islam, laïcité, démocratie », Pouvoirs, n²104, p. 5-19.

FLORI J., 2002, Guerre sainte, jihad, croisade. Violence et religion dans le christianisme et l'islam, Paris, Le Seuil, $342 \mathrm{p}$.

FOUDA Y., FIELDING N., 2005, Les cerveaux du terrorisme, Paris, Éditions du Rocher, $385 \mathrm{p}$.

FOUREST C., 2001, Foi contre choix. La droite religieuse et le mouvement «prolife» aux États-Unis, Villeurbanne, Golias, $334 \mathrm{p}$.

— VENNER F., 2005 [2003, Calmann-Lévy], Tirs croisés. La laïcité à l'épreuve des intégrismes juif, chrétien et musulman, Paris, Livre de Poche, $543 \mathrm{p}$.

FRACHON A., VERNET D., 2004, L'Amérique messianique, Paris, Le Seuil, 240 p.

FUENTES C., 2004, Contre Bush, Paris, Gallimard, 224 p.

GEISSER V., 2003, La nouvelle islamophobie, Paris, La Découverte, 128 p. 
GLUCKSMANN A., 2004, Le discours de la haine, Paris, Plon, 235 p.

- 2003, Ouest contre Ouest, Paris, Plon, $250 \mathrm{p}$.

GOLDZIHER I., 2003 [rééd.], Sur l'Islam. Origines de la théologie musulmane, Paris, Desclée De Brouwer, $290 \mathrm{p}$.

GOODY J., 2004, L'islam en Europe. Histoire, échanges, conflits, Paris, La Découverte, $180 \mathrm{p}$.

GOZLAN M., 2002, Pour comprendre l'intégrisme islamiste, Paris, Albin Michel, $199 \mathrm{p}$.

GRESH A., 2004, L'islam, la République et le monde, Paris, Fayard, 432 p.

HABERMAS J., 2002, L'avenir de la nature humaine, Paris, Gallimard, $192 \mathrm{p.}$

HEISBOURG F., 2003, Hyperterrorisme : la nouvelle guerre, Paris, Odile Jacob, $270 \mathrm{p}$.

IOGNA-PRAT D., VEINSTEIN G., 2003, Histoire des hommes de Dieu dans le christianisme et l'islam, Paris, Flammarion, $298 \mathrm{p}$.

JACQUARD R., 2001, Au nom d'Oussama Ben Laden..., Paris, Jean Picollec, 400 p.

— 2002, Les archives secrètes d'Al Qaida, Paris, Jean Picollec, $367 \mathrm{p}$.

- TAZAGHART A., 2004, Ben Laden. La destruction programmée de l'Occident, Paris, Saint-Simon, $509 \mathrm{p}$.

JULLIARD J., 2003, Rupture dans la civilisation. Le révélateur irakien, Paris, Gallimard, $92 \mathrm{p}$.

KAHN J.-F., 2004, Le camp de la guerre. Critique de la déraison impure, Paris, Fayard, $283 \mathrm{p}$.

KALTENBACH J.-H., TRIBALAT M., 2002, La République et l'islam. Entre crainte et aveuglement, Paris, Gallimard, $338 \mathrm{p}$.

KEPEL G., 2002 [1991], Chronique d'une guerre d'Orient, Paris, Gallimard, 130 p.

- 2003, La revanche de Dieu. Chrétiens, juifs et musulmans à la reconquête du monde, Paris, Le Seuil (Points), $289 \mathrm{p}$.

— 2002 [2000], Jihad. Expansion et déclin de l'islamisme, Paris, Gallimard (Folio actuel), $750 \mathrm{p}$.

— 2003, "Jihad », Pouvoirs, $\mathrm{n}^{\circ}$ 104, Islam et démocratie, p. 135-142.

- 2004, Fitna. Guerre au cœur de l'islam, Paris, Gallimard, 400 p.

KHOROSKHAVAR F., 2003, Les nouveaux martyrs d'Allah, Paris, Flammarion, $369 \mathrm{p}$.

— 2004, L'islam dans les prisons, Paris, Balland, 286 p.

KISSINGER H., 2003, La nouvelle puissance américaine, Paris, Fayard, 507 p.

KRUGMAN P., 2004, L'Amérique dérape, Paris, Flammarion, 352 p.

LA BRANCHE S., 2003, Mondialisation et terrorisme identitaire ou comment l'Occident tente de transformer le monde, Paris, L'Harmattan (Logiques sociales), $284 \mathrm{p}$.

LACORNE D., 2003 [1997, Fayard], La crise de l'identité américaine. Du melting-pot au multiculturalisme, édition revue et augmentée, Paris, Gallimard, 448 p.

LAÏDI A. (en collaboration avec AHMED S.), 2002, Le jihad en Europe. Les filières du terrorisme islamiste. Paris, Le Seuil (L'épreuve des faits), $290 \mathrm{p}$.

LAMCHICHI A., 2001, Géopolitique de l'islamisme, Paris, L'Harmattan, 334 p.

— 2004, "Islam(s) et islamisme(s) à l'épreuve de la violence et de la guerre », H. Lelièvre éd. (v. infra), Terrorisme: questions, p. 73-109.

LANDAU P., 2005, Le Sabre et le Coran. Tariq Ramadan et les Frères musulmans à la conquête de l'Europe, Paris, Éditions du Rocher, 236. p.

LAURENTÉ., 2003, Le monde secret de Bush. La religion, les affaires, les réseaux occultes, Paris, Plon, $218 \mathrm{p}$.

LÉGER J.-A., 2003, Tartuffe fait ramadan, Paris, Denoël, 140 p. 
- 2004, À contre-Coran, Paris, Hors-commerce, $164 \mathrm{p}$.

LELIÈVRE H. éd., 2004, Terrorisme : questions, Bruxelles, Complexe, $264 \mathrm{p}$.

LENOIR F., 2004, Les métamorphoses de Dieu. La nouvelle spiritualité occidentale, Paris, Plon, $404 \mathrm{p}$.

L'Express, 13-19 septembre 2001 et 20-26 septembre 2001.

Le Nouvel Observateur, 24 décembre 2003-7 janvier 2004 (numéro double), La Bible et le Coran. 48 pages pour éclairer les déchirements de notre histoire et les enjeux d'aujourd'hui.

- 21-27 avril 2005, Sur la route des croisades. Aux sources du conflit entre l'Occident et l'Orient.

L'Histoire, $\mathrm{n}^{\circ}$ 259, novembre 2001, Le dossier noir de l'Inquisition.

- n²60, décembre 2001, La vérité surl'islam.

- $n^{\circ} 272$, janvier 2003, Les Arabes, de La Mecque aux banlieues de l'islam.

- $n^{\circ} 281$, novembre 2003, Les islamistes.

- $\mathrm{n}^{\circ} 284$, février 2004, Les racines de la droite américaine.

— n²89, juillet-août 2004, Dieu et la politique. Le défi laïque.

LEWIS B., 2005, Islam, Paris, Gallimard, 1344 p.

MAILER N., 2003, Pourquoi sommes-nous en guerre?, Paris, Denoël, 113 p.

MANN M., 2004, L'Empire incohérent, Paris, Calmann-Lévy, $384 \mathrm{p}$.

MARRET J.-L. éd., 2005, Les fabriques du jihad, Paris, PUF, 335 p.

MARTINEZ-GROS G., VALENSI L., 2004, L'islam en dissidence. Genèse d'un affrontement, Paris, Le Seuil, $336 \mathrm{p}$.

MEDDEB A., 2002, La maladie de l'islam, Paris, Le Seuil, 223 p.

- 2004, Face à l'islam (entretiens), Paris, Textuel, $224 \mathrm{p}$.

MÉNORET P., 2003, L'énigme saoudienne. Les Saoudiens et le monde, 1744-2003, Paris, La Découverte, $260 \mathrm{p}$.

MÉRAD A., 2001, La tradition musulmane, Paris, PUF (Que sais-je ?), 128 p.

— 2002 [1984], L'Islam contemporain, 6e édition mise à jour, Paris, PUF (Que sais-je), $128 \mathrm{p}$.

MERIBOUTE Z., 2004, La fracture islamique: demain, le soufisme?, Paris, Fayard, 384 p.

MILLIERE G., 2004, Pourquoi Bush sera réélu, Paris, Michalon, 162 p.

MOORE M., 2004, Mike contre-attaque. Bienvenue aux États Stupides d'Amérique, Paris, 10/18, $224 \mathrm{p}$.

MOUTTAPA J., 2001, «Un terme piégé : le djihad », Actualité des religions, n 6, 2001.

NASR M., 2001, Les Arabes et l'islam vus par les manuels scolaires français (1986 et 1997), Paris, Karthala, $376 \mathrm{p}$.

OCKRENT C., 2004, Bush-Kerry, les deuxAmérique, Paris, Laffont, 299 p.

PEARL M., 2003, Un cœur invaincu, Paris, Plon, $350 \mathrm{p}$.

Pouvoirs, 2003, $\mathrm{n}^{\circ} 104$, Islam et démocratie, $203 \mathrm{p}$.

PRIMOR A., 2004, Du terrorisme et de ceux qui l'exploitent, Paris, Bayard, 273 p.

Projet, juillet 2004, $\mathrm{n}^{\circ} 281$ (spécial), Religions et violence, $94 \mathrm{p}$.

QUANTIN J.-L., 2001, Le rigorisme chrétien, Paris, Cerf, 168 p.

Question de, $1^{\text {er }}$ trimestre 2002, Pour un islam de paix, $262 \mathrm{p}$.

RAMADAN T., 2003, Les musulmans d'Occident et l'avenir de l'islam, Paris, Sindbad Actes

Sud, $383 \mathrm{p}$.

RANDAL J., 2004, Oussama, fabrication d'un terroriste, Paris, Albin Michel, 415 p.

RASHID A., 2001, L'ombre des Talibans, Paris, Autrement. 
RAZAVI E., 2005, Frères musulmans dans l'ombre d'Al Qaeda, Paris, Jean-Cyrille Godefroy, $223 \mathrm{p}$.

REDISSI H., 2004, L'exception islamique, Paris, Le Seuil, 238 p.

RÉMOND R., 2001, Religion et société en Europe, Paris, Le Seuil, 307 p.

REYMOND W., 2004, Bush land (2000-2004), Paris, Flammarion, $453 \mathrm{p}$.

RICHETI., 2003, La religion aux États-Unis, Paris, PUF (Que sais-je ?), 128 p.

RODINSON M., 2003 [1980], La fascination de l'islam, Paris, La Découverte, 200 p.

ROLLET J., 2001, Religion et politique. Le christianisme, l'islam, la démocratie, Paris, Grasset, $287 \mathrm{p}$.

ROUGIER B., 2004, Le jihad au quotidien, Paris, PUF, 262 p.

ROY 0., 2002, L'islam mondialisé, Paris, Le Seuil, 240 p.

— 2002, Généalogie de l'islamisme, Paris, Hachette, 118 p.

- 2002, Les illusions du 11 septembre. Le débat stratégique face au terrorisme, Paris, Le Seuil, $86 \mathrm{p}$.

SAGEMAN M., 2005, Le vrai visage des terroristes. Psychologie et sociologie des acteurs du djihad, Paris, Denoël, $396 \mathrm{p}$.

SCHLEGEL J.-L., 2003, La loi de Dieu contre la liberté des hommes. Intégrismes et fondamentalismes, Paris, Le Seuil, $142 \mathrm{p}$.

SEDDIKY., 2004, Nous n'avons jamais lu le Coran, Paris, Éditions de l'Aube, 298 p.

SESBOÜÉ B., 2004, Hors de l'Église pas de salut. Histoire d'une formule et problèmes d'interprétation, Paris, Desclée de Brouwer, $396 \mathrm{p}$.

SFEIR A., 2001, Les réseaux d'Allah. Les filières islamistes en France et en Europe, la nébuleuse Ben Laden, Paris, Plon, $271 \mathrm{p}$.

SIFAOUI M., 2002, La France malade de l'islamisme. Menaces terroristes sur l'Hexagone, Paris, Le cherche midi, $216 \mathrm{p}$.

— 2003, Mes «frères » assassins. Commentj'ai infiltré une cellule d'Al-Qaïda, Paris, Le cherche midi, $180 \mathrm{p}$.

- 2004, Sur les traces de Ben Laden, Paris, Le cherche midi, $189 \mathrm{p}$.

Spiritus, $\mathrm{n}^{\circ} 171$, juin 2003, La tentation fondamentaliste.

SUSKIND R., 2003, Le roman noir de la Maison-Blanche, Paris, Saint-Simon, $461 \mathrm{p}$.

TAHA M., 2002, Un islam à vocation libératrice, Paris, L'Harmattan, $180 \mathrm{p}$.

TERNISSIEN X., 2004 [2001], La France des mosquées, édition revue et augmentée, Paris, Albin Michel, $303 \mathrm{p}$.

— 2005, Les Frères musulmans, Paris, Fayard, 367 p.

THOMAS D., 2005, Les hommes d'Al-Qaïda. Discours et stratégie, Paris, Michalon, $120 \mathrm{p}$. TISSIER de MALLERAIS B., 2002, Marcel Lefèbvre, une vie, Paris, Clovis, $719 \mathrm{p}$.

TOLAN J., 2003, Les Sarrazins. L'islam dans l'imagination européenne au Moyen Âge, Paris, Aubier, $478 \mathrm{p}$.

VALLET 0., 2003, Les religions dans le monde, Paris, Flammarion, $154 \mathrm{p}$.

- 2004, Petit lexique des guerres de religion d'hier et d'aujourdhui, Paris, Albin Michel, $161 \mathrm{p}$.

VAN NISPEN TOT SEVENAER C., 2004, Chrétiens et musulmans, frères devant Dieu ?, Paris, L'Atelier, $189 \mathrm{p}$.

VICTORIA B., 2001, Le zen en guerre, traduit de l'anglais (Zen at War) par Luc Boussard, Paris, Le Seuil, $370 \mathrm{p}$. 
VENNER F., 2005, OPA sur l'islam de France. Les ambitions de l'UOIF, Paris, Calmann-Lévy, $247 \mathrm{p}$.

VICTOR B., 2004, La dernière croisade. Les fous de Dieu, version américaine, Paris, Plon, $347 \mathrm{p}$.

WIEVIORKA M. éd., 2004, L'Empire américain ?, Paris, Balland, $279 \mathrm{p}$.

WOODWARD B., 2003, Bush s'en va en guerre, Paris, Denoël, 382 p.

— 2004, Plan d'attaque, Paris, Denoël, 473 p.

YACOUB J., Au nom de Dieu! Les guerres de religion d'aujourdhui et de demain, Paris, Lattès, $308 \mathrm{p}$.

ZANCARINI J.-C., 2003, "À propos du bien et du mal ou Machiavel versus Bush », Mots. Les Langages du politique, $\mathrm{n}^{\circ} 73$, Les discours de la guerre, p. 169-172.

ZARCA Y.-Ch., TAUSSIG S., FLEURY C. éd., 2004, Cités, hors série, L'islam en France, Paris, PUF, $352 \mathrm{p}$.

ZARCONE T., 2004, La Turquie moderne et l'islam, Paris, Flammarion, 362 p. 